\title{
Measurement System of the Relative Humidity With Capacitive Sensor by CORECI 5000
}

\author{
Monica-Anca Chita ${ }^{1 *}$ \\ ${ }^{1}$ University of Pitesti, Pitesti, 110040, Romania. \\ *chita_monica@yahoo.com and monica.chita@upit.ro
}

\begin{abstract}
In this paper is presented a circuit for the relative humidity measurement. A humidity capacitive sensor of type HUMICOR 5000 (produced of CORECI firm) is used. This sensor transforms the humidity variations into capacitance variations. The measurement principle consists in the inclusion of sensor in a UA 555 oscillator, of which output frequency is dependent on the relative humidity. A frequency-voltage converter with Phase Locked Loop (PLL) is used for obtain a voltage dependent to relative humidity. The PLL using for the frequency-voltage conversion is a simple solution, which presents the advantage of a very low sensibility at noise that can affect the input signal. The simplicity of the proposed measurement circuit and the obtained results, a series of experimental results obtained by measurement and SPICE simulation presented in the paper, allows his utilisation in different applications such as environment monitoring, pharmaceutical industry, tile and brick drying, etc.
\end{abstract}

\section{Introduction}

The numerous fabrications processes of a product require the knowledge of the air or a gas humidity degree (or the humidity absence (dryness)) [1]. The necessity of the relative humidity knowing in different technological process determined an ample development of the humidity sensors [2]. A solution in the capacitive humidity sensors realisation is the polymer use as the dielectric. Thus, certain polymers constitute excellent dielectrics and allow the realisation of some sensors sensitive at the relative humidity (for climatic maintenance by example) [3]. The response of this type of sensors is almost linear and his utilisation into an oscillator makes that the output voltage frequency to be dependent of the humidity.

CORECI sensors allow the directly relative humidity measurement. Thus the relative humidity variation is transform in a sensor capacity variation. The operating principle is based on the hygroscopic material used as the dielectric with a rapid adaptive and reversible capacitor at the environment humidity and having a strong variation of capacity. The sensors and their components have been specially designed and manufactured to provide excellent metrological features while avoiding interference of the measured environment. These sensors feature immediate response and reduce hysteresis. It can be used under repetitive and prolonged conditions of humidity (close to saturation). Condensation or wetting do not alter the sensors operating capabilities.

\section{The Sensor Structures and Characteristics}

The HUMICOR 5000 [4] used sensor, of which structure is presented in Fig. 1, is formed from an glass substrate, a lower electrode of tantalum fall out through thermal evaporation, an upper electrode realised from a chrome film, a dielectric from synthetic polymer and a protection film.

The main characteristics of the sensor are presented in the Tables 1 and 2, where \%RH represents the relative humidity in percents. The sensors response time represents the period of time necessary for a $90 \%$ final capacity variation. 


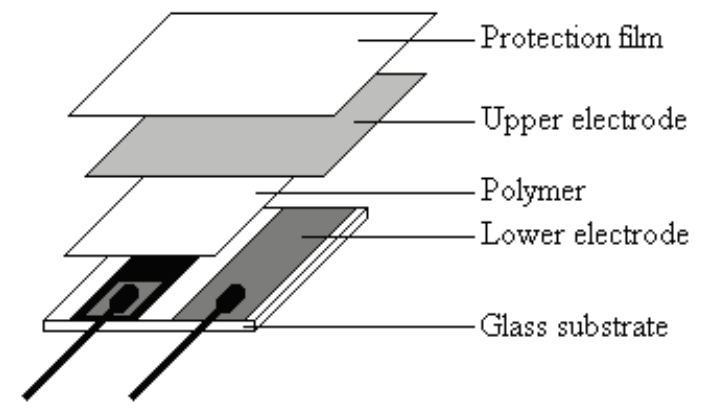

Figure 1. The structure of the sensor.

\section{The Presentation of the Relative Humidity Measurement Circuit with Capacitive Sensor}

In Fig. 2 is presented the block diagram of the relative measurement circuit, including the analogdigital converter block (ADC) necessary for the coupling to a PC.

The sensor is a capacitor which capacity depends of the relative humidity.

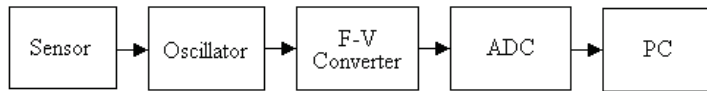

Figure 2. The block diagram of the measurement system of relative humidity.

The oscillator provides at the output a frequency depending of the relative humidity. The output oscillator can be coupled directly to the next block or can be applied at the transmission line input, when the signal transmission at the distance is necessary.

The output voltage of frequency-voltage converter is in proportion with oscillator frequency. This voltage can be applied directly to a voltmeter, or to an analog-digital converter which is connected to a PC.

The oscillator is realised using the delay circuit UA555, like in Fig. 3, configured at symmetric astable multivibrator. Using the diodes D1-D4, a duty cycle equal to 0.5 it is obtained. If the capacitance value of $\mathrm{C} 1$ increases, than the frequency-humidity characteristic is displaced down. Optimal value of $\mathrm{C} 1$ was determined by
SPICE simulation of circuit model, in two situations. First, if the measurement circuit is coupled to a voltmeter, than a high precision in the range $10-70 \% \mathrm{RH}$ it is important. Determined optimal value in this case is $\mathrm{C} 1=16 \mathrm{nF}$. Second, the measurement circuit is coupled to data acquisition system, than it is important that the oscillator frequency range must be precisely, in which case a data acquisition processing can compensate the non-linear characteristic. The requisite oscillator frequency range is $50-60 \mathrm{kHz}$, for a sensor capacity range 476-576.6 pF, corresponding at $100 \% \mathrm{RH}$ to $0 \% \mathrm{RH}$ variation. In this condition it is obtained $\mathrm{Cl}=30 \mathrm{nF}$.

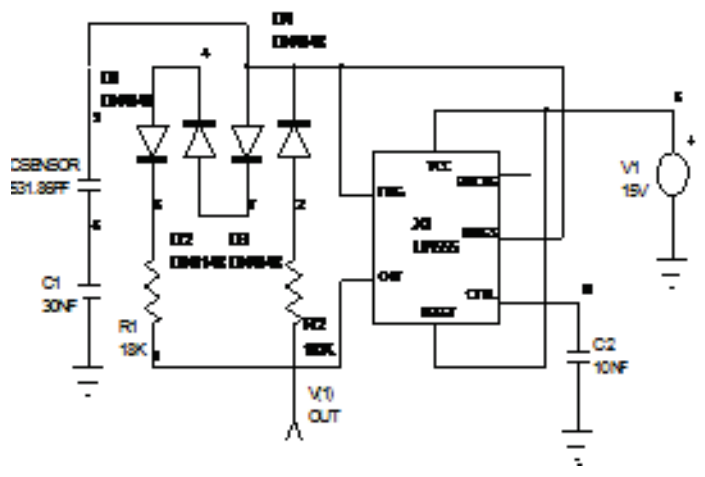

Figure 3. The oscillator.

The frequency-voltage converter is presented in Fig. 4. This circuit provides to output a voltage between $0 \mathrm{~V}$ and $5 \mathrm{~V}$ for an input frequency between $60 \mathrm{kHz}$ and $50 \mathrm{kHz}$. Thus, $0 \% \mathrm{RH}$ corresponds to $0 \mathrm{~V}$ at the output converter and 100 $\% \mathrm{RH}$ corresponds to $5 \mathrm{~V}$.

The utilisation of the Phase Locked Loop (PLL) for frequency-voltage conversion is a simple solution. Note that PLL has a low sensibility to input noise. This advantage is important especially when the oscillator and the converter are connected through a long transmission line.

For the Voltage Controlled Oscillator (VCO) design, following relationships are used [5].:

$$
f_{\text {min }}=\frac{1}{R_{2} \cdot\left(C_{1}+32 p F\right)}
$$




$$
f_{\max }=\frac{1}{R_{1} \cdot\left(C_{1}+32 p F\right)}+f_{\min }
$$

where fmin $=50 \mathrm{kHz}$ and fmax $=60 \mathrm{kHz}$ represent the limits of $\mathrm{f}-\mathrm{v}$ converter range. The resistors Rp1 and Rp2 allow calibrating the limits of range frequency, fmin and fmax, for PLL.

The nominal values of low pas filter (LPF), used for loop filter, are obtained on condition that the PLL natural frequency $\left(\omega_{\mathrm{n}}\right)$ be 10 times less than its center frequency (f0) [6]:For the Voltage Controlled Oscillator (VCO) design, following relationships are used [5]:

$$
\omega_{n}=\frac{2 \cdot \pi \cdot f_{0}}{10}
$$

where $\mathrm{f}_{0}=55 \mathrm{kHz}$ and $\omega_{\mathrm{n}}$ can be obtained with the following relationship:

$$
\omega_{n}=\sqrt{\frac{K_{\phi} \cdot K_{O C T}}{R_{3} \cdot C_{2}}}
$$

The phase comparator (PC2) gain is [5]:

$$
K_{\phi}=\frac{V_{D D}}{4 \cdot \pi}
$$

The VCO gain is [5]:

$$
K_{O C T}=\frac{2 \cdot \pi \cdot \Delta f_{O C T}}{V_{D D}-2}
$$

The recommended table Using this relationship, the value for the external components of the VCO and for the components of the LPF are calculated and marked in Fig. 4.

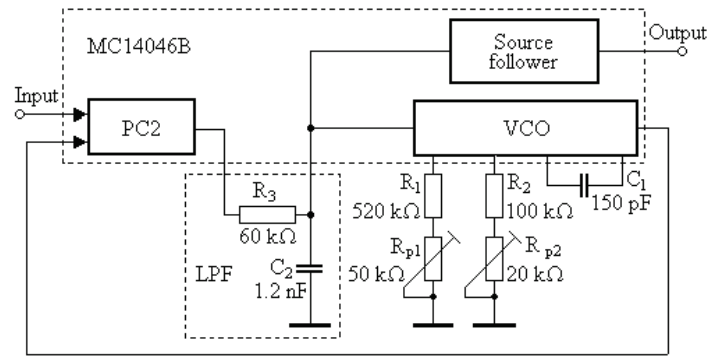

Figure 4. Frequency-voltage converter with PLL.4 Experimental Results and Simulation
The measures were effected for the relative humidity control in eleven points, using eleven bottles containing saturation saline solutions of chloride of lithium [7].

The capacity measurement of the relative humidity capacitive sensor was make with a digital RCL-meter of type PM 6401.

The values of sensor capacity obtained are presented in second column of the Table 3 (in the third column of the table being presented the results obtained from mathematical modelling of sensor capacity (relation 7)) and graphically represented in Fig. 5, using the facilities offered of the MATLAB language.

Table 1. Experimental results and obtained by modelling.

\begin{tabular}{|c|c|c|}
\hline $\begin{array}{c}\text { Relative } \\
\text { humidity [\%RH] }\end{array}$ & $\begin{array}{c}\text { Experimental } \\
\text { capacitance }[\mathrm{pF}]\end{array}$ & $\begin{array}{c}\text { Modeled } \\
\text { capacitance }[\mathrm{pF}]\end{array}$ \\
\hline 0 & 474 & 476.00 \\
\hline 10 & 490 & 491.80 \\
\hline 20 & 501 & 503.10 \\
\hline 30 & 511 & 513.10 \\
\hline 40 & 521 & 522.62 \\
\hline 50 & 531 & 531.86 \\
\hline 60 & 540 & 540.95 \\
\hline 70 & 549 & 549.95 \\
\hline 80 & 560 & 558.88 \\
\hline 90 & 569 & 567.77 \\
\hline 100 & 579 & 576.62 \\
\hline
\end{tabular}

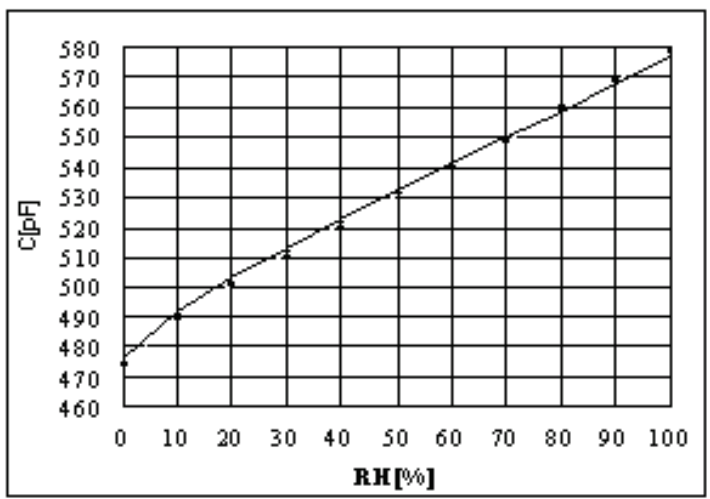

Figure 5. The dependence $\mathrm{C}=\mathrm{C}(\mathrm{RH})$, where $\mathrm{x}$ are the experimental points, and - is modelling. 
The HUMICOR 5000 sensor response signal has been modelled and it is indicated for one point calibration by the equation:

$$
C=\left(a \cdot R H-\frac{b}{R H+c}+d\right) \cdot C 75
$$

in which: $\mathrm{a}=1,56910-3, \mathrm{~b}=0,298, \mathrm{c}=11, \mathrm{~d}=$ 0,88475 ; $\mathrm{C}$ is the sensor capacity value in $\mathrm{pF}$; $\mathrm{RH}$ is the humidity value on sensor location (\%RH); C75 is the indicated capacity value (at $75 \% \mathrm{RH}$ ) of each sensor.

Using the mathematical model for sensor and the two values of the capacitor $\mathrm{C} 1$, it was obtained by the SPICE simulation of the oscillator, the results from Table 2. In Fig. 6 is presented the oscillator frequency versus relative humidity.

Table 2. Results obtained by modelling

\begin{tabular}{|c|c|c|}
\hline $\begin{array}{c}\text { Relative } \\
\text { humidity }[\% \mathrm{RH}]\end{array}$ & $\begin{array}{c}\text { Frequency }\left(\mathrm{C}_{1}=\right. \\
16 \mathrm{nF})[\mathrm{kHz}]\end{array}$ & $\begin{array}{c}\text { Frequency }\left(\mathrm{C}_{1}=\right. \\
30 \mathrm{nF})[\mathrm{kHz}]\end{array}$ \\
\hline 0 & 60.97 & 59.88 \\
\hline 10 & 58.82 & 58.00 \\
\hline 20 & 57.80 & 56.95 \\
\hline 30 & 56.82 & 55.99 \\
\hline 40 & 55.61 & 55.01 \\
\hline 50 & 54.81 & 54.05 \\
\hline 60 & 54.05 & 53.47 \\
\hline 70 & 53.19 & 52.63 \\
\hline 80 & 52.51 & 51.54 \\
\hline 90 & 51.81 & 51.02 \\
\hline 100 & 51.02 & 50.25 \\
\hline
\end{tabular}

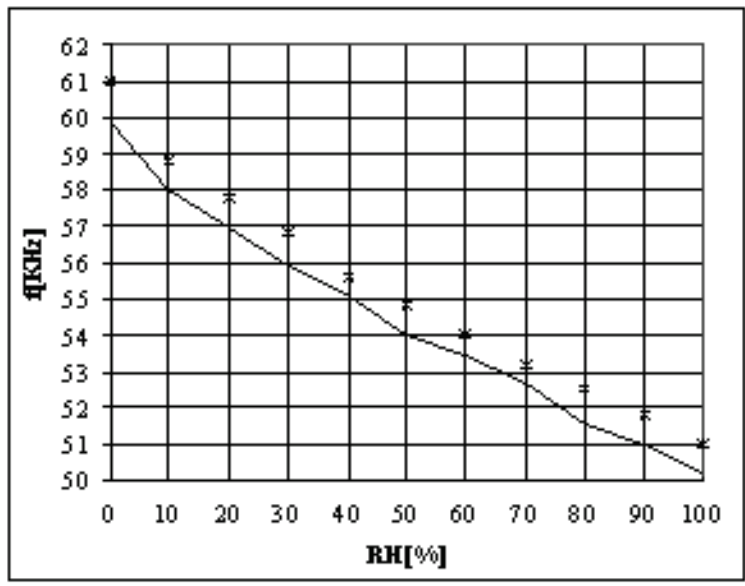

Figure 6. The dependence $f=f(R H)$, where:

$$
\mathrm{x}_{1}=16 \mathrm{nF} ; \quad-\mathrm{C}_{1}=30 \mathrm{nF}
$$

The simulation results of whole circuit are presented in Table 3 and Fig. 7.

Table 3. Results obtained by simulation of $\mathrm{RH}$ measurement circuit.

\begin{tabular}{|c|c|c|}
\hline $\begin{array}{c}\text { Relative } \\
\text { humidity [\%RH] }\end{array}$ & $\begin{array}{c}\mathrm{V}_{0}\left(\mathrm{C}_{1}=16 \mathrm{nF}\right) \\
{[\mathrm{V}]}\end{array}$ & $\begin{array}{c}\mathrm{V}_{0}\left(\mathrm{C}_{1}=30 \mathrm{nF}\right) \\
{[\mathrm{V}]}\end{array}$ \\
\hline 0 & 5.48 & 4.94 \\
\hline 10 & 4.41 & 4.00 \\
\hline 20 & 3.90 & 3.47 \\
\hline 30 & 3.41 & 2.99 \\
\hline 40 & 2.80 & 2.50 \\
\hline 50 & 2.40 & 2.03 \\
\hline 60 & 2.03 & 1.73 \\
\hline 70 & 1.56 & 1.32 \\
\hline 80 & 1.25 & 0.77 \\
\hline 90 & 0.90 & 0.51 \\
\hline 100 & 0.51 & 0.13 \\
\hline
\end{tabular}

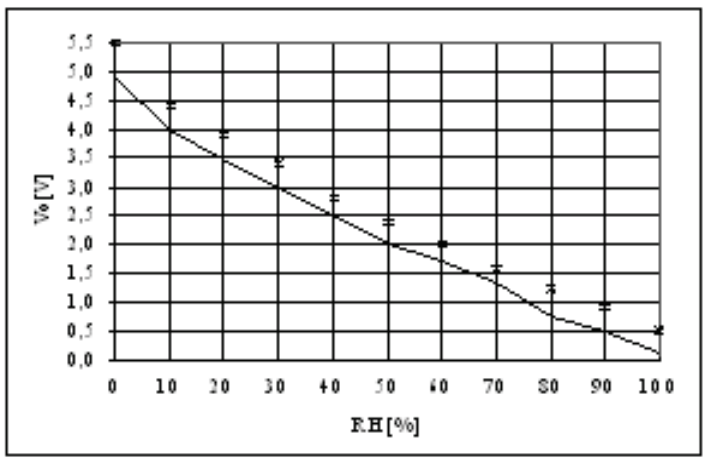

Figure 7. The dependence $\mathrm{Vo}=\mathrm{Vo}(\mathrm{RH})$, where:

$$
\mathrm{x}_{1}=16 \mathrm{nF} ; \quad-\mathrm{C}_{1}=30 \mathrm{nF}
$$

We observe that, like we saw in section 2, for a high measurement precision in the range 10-70 $\% \mathrm{RH}$, it is useful to get up the voltage- $\mathrm{RH}$ characteristic by decreasing the capacity value of $\mathrm{C} 1$. If $\mathrm{C} 1=30 \mathrm{pF}$ than it obtains a high precision for relative humidity measurement at range extremity, but in the middle of the range it obtains a low precision.

A good convergence of whole circuit simulation was obtained using the Gear method. For PLL simulation it used the APLL macromodel presented in [8]. 


\section{Conclusions}

The HUMICOR 5000 sensors are capacitive humidity sensors, providing a fast and accurate measurement of relative humidity in air, gases and other products. These sensors are suitable for most applications and difficult environmental conditions: high humidity level, wide temperature range, repetitive condensation or wetting.

The use of the relative humidity capacitive sensor HUMICOR 5000 allows the fast measurement (his response time is very small, only $2 \mathrm{~s}$ ) and enough exactly of the relative humidity in very extremely different environmental conditions.

Because has a very small mass $(0.1 \mathrm{~g})$ the relative humidity capacitive sensor HUMICOR 5000 practical not disturb the medium in which it make the measurement, knowing the fact that the passive sensors, through him simple presence into the measurement precincts, disturb between biggest or smallest measure the medium in which have the measurement. Thus it can be realised the measurements of big fineness using such sensor.

The HUMICOR response curve illustrates the large sensitivity range of the capacity for $0-100$ $\% \mathrm{RH}$ measurement range, that is a typical variation of approximately $100 \mathrm{pF}$ for $100 \% \mathrm{RH}$ variation.

The used oscillator is a sensor's capacityfrequency converter which sensitivity is 100 $\mathrm{Hz} / \% \mathrm{RH}$. This oscillator is easy to build but it presents a non-linear characteristic. This disadvantage can be compensated by an optimal choice of $\mathrm{C} 1$ value. Thus it obtains a better accuracy than $\pm 2.5 \%$ but only in range 10-70 $\% \mathrm{RH}$. The optimal value of $\mathrm{C} 1$ was determined by SPICE simulation of the oscillator. For the practical case, the oscillator can be calibrate for a known RH, for example at $50 \% \mathrm{RH}$.

The PLL has a low sensitivity to noise and to perturbation signals which are added to input signal. This important advantage was determined the choice of converter.

The simplicity of proposed measurement circuit and the obtained results recommend its using in many applications (such as environment monitoring, fruit and vegetable storage, etc.).

\section{References}

1) I. Karube, Handbook of Measurement Science, Vol. III, (Edited by Synderham,London, 1992), 124.

2) Z. Gotta, K. Kamuda, J. Potencki, Higly sensitive microelectronics sensor for measurement of physical quantities with increased response dynamic, The 5th International Symposium for Design and Technology in Electronic Modules SIITME'99, September, 23rd-26th 1999, Bucharest, Romania, pp. 57-61.

3) H. Grange, G. Delapierre, Polymer-based capacitive higrometers, (Edited by Cedex, Grenoble, 1995), pp 256.

4) $* * *$, CORECI documentation, 2014.

5) $* * *$, Semiconductor Technical Data, Motorola, 1997, http://motorola.com/sps/.

6) Nash, Phase Locked Loop Design Fundamentals, AN535, Motorola,

7) .M.A. Chita, M. Raducu, I. Popa, Measurement system of the relative humidity with capacitive sensor externally connectable at a $P C$, The 23nd International Spring Seminar on Electronics Technology, Conference Proceedings ISSE 2000, May 610, Balatonfüred, Hungary, 2000, pp. 143147 ,.

8) M. Raducu, M.A. Chita, I. Popa, A SPICE Modeling for Analog Phase-Locked Loop, The 25th Congress of the American Romanian Academy of Arts and Sciences (ARA), Cleveland, Ohio, USA, July 12-16, 2000, Proceedings, pp. 256-259, 